\title{
Computer-assisted Three-Dimensional Sports Training System Analysis and Design Research
}

\author{
ZHOU LI ${ }^{1, \text { a }}$ \\ ${ }^{1}$ Physical Education, Jiangxi Science and Technology Normal University, Nanchang 330013,China \\ azhoulijx@163.com
}

Keywords: Computer-Assisted Three-Dimensional; Sports Training; System Analysis

\begin{abstract}
. sports simulation helps sports training, the transition from primarily based on the human eye's method to the traditional method based on anthropometric high-definition video capture and analysis; from experience-based training methods to the stylized sports science shift analysis method, leading to faster and more effectively improve the level of sports training and athletic performance. Computer simulation sport in sports field has broad application prospects in recent years, it has been successfully applied, such as diving, gymnastics, trampoline, weightlifting and other competitive sports. By summing sports simulation modeling, analyzing the characteristics and scope of several main modeling methods, to further discuss the sports simulation visualization techniques, prospects of its future development trend.
\end{abstract}

\section{Introduction}

Due to the development of computer technology, to increase data storage capacity and price decline rapidly, causing data to be stored sport athletes is a quick and cheap to do. How to use these large amounts of data, in-depth analysis of technical movements essentials, in the training available to athletes and coaches are very useful reference information [3]. Therefore, the application of modern computer technology has become one of the most important tools to enhance the level of competition. Combining years of research experience, for a regular sports action, computer-based three-dimensional processing technology, combined with regular physical activity build sports technical movement features data model, mining association rules between action sports technology, to improve the competitive level of athletes, providing a scientific research methods, has a relatively good reference value.

\section{Human joint model overview}

Data can come from human joints motion capture system data (motion capture system) or Microsoft Kinect depth camera obtained after processing. After getting the data key point is how to build a three-dimensional human body model. Human skeleton model is the foundation for the human body model. Because it contains the main points of the joint three-dimensional location information, it can better represent the human information. As used herein human joint model shown in Figure 1, it contains a total of 23 joints, the waist of the root node. Because of the resulting motion capture coordinate data is based on the world coordinate system, we first will coordinate every action will convert to the root of the coordinate origin of the world coordinate system, in order to make the action sequence data regardless of the absolute position of the body [4-5]. 


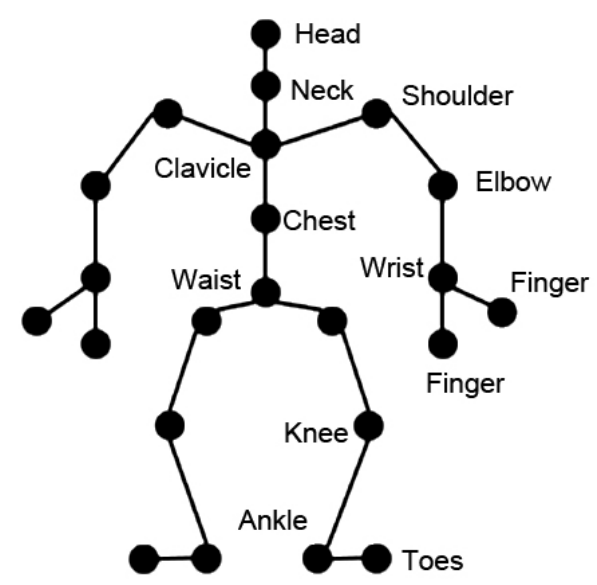

Figure1. 3D human skeleton model

At the same time, also the body toward the initial positioning z-axis positive direction so that the initial orientation has nothing to do with human data. Meanwhile, we will get different people human skeleton length normalized to the same skeleton size to make size has nothing to do with the human body motion data.

This can be unified into a standard 23-point coordinates joint, effective data dimension of 67-dimensional (height information included the root of the cross-sectional information is 0 ). We will also be broken down into a series of human skeleton parts, each part is a combination of single joints or joint points. Such node classification contains semantic information, so we are more effectively extracted to identify the critical part of the operation.

\section{Build sports technical movement features of the model}

Kinect combination of the above analysis and human joint model of technical action design, and in order to be able to quickly and easily build sports action feature model, the need for action by decomposition and activity classification, to achieve the Players package. Movements of the database is to achieve action feature model key supporting technologies. As a general unified basic tools, action library should have completeness, independence, versatility features [6].

In order to achieve completeness and independence movement database design requirements, we need to realize the proper classification of technical movements; but in order to achieve common requirements, we need to ensure that action library is scalable. In accordance with the aforementioned technical action sports action task decomposition model and classification methods, we propose a hierarchical structure athlete action library, from top to bottom can be broadly classified into action unit layer, layer, action elements and technical operation layer, as shown in Figure 2.

The basic action elements layer: This layer contains only one basic motilin unit. The basic elements of the action framework defines the basic action sports athletes, and describes some of the basic elements of relevant, including the start-up operation cycle, pause and stop, and general motion parameters.

Technical action elements layer: technical action elements obtained from the basic operation of the unit derived elements, with the most basic semantic features sports action, but the action can not be subdivided semantically, is the most basic units of human motion sports action. Sports action elements generally follow the model of human joints and freedom of movement to divide.

Action unit layer: technical action unit is composed of several technical action elements constituted according to certain semantic rules, with a certain degree of semantic features, describes the technical operation of semantic features common sense, but does not include any specific activities related to the operation semantics.

In action basic layer, we based on the limited state design is a basic action basic classes, the class action elements are elements of all the actions and action unit of the parent class, is responsible for implementing the technical movement of cyclic scheduling; Elements layer in the 
movement, the movement basic derived and encapsulating the plane movement, attitude adjustment, unarmed, tool operation such as dynamic operation element unit; Cell layer in the movement, based on the action in the further derived from the action elements with a specific semantic features of action unit.

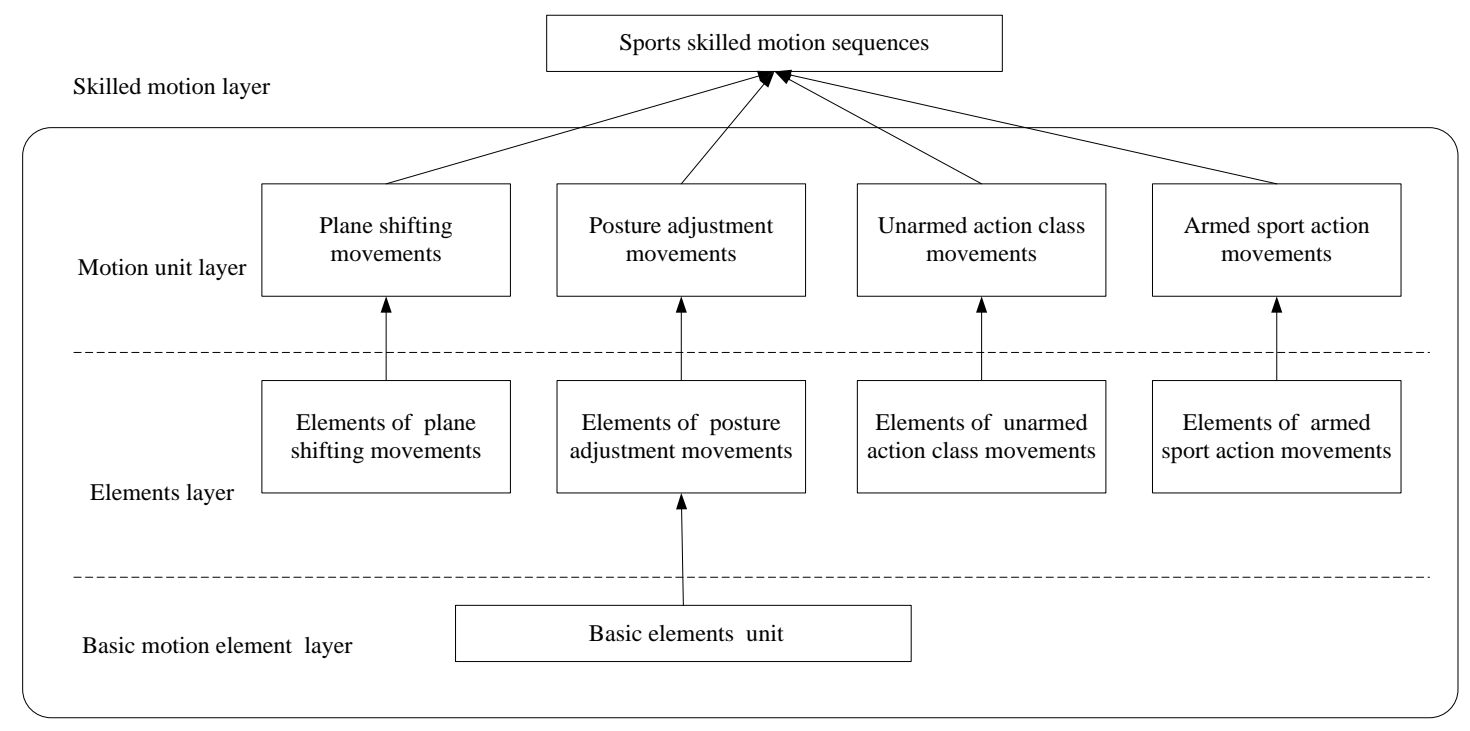

Figure 2.The hierarchical model of sports skilled motion characteristic

\section{D Sport Training Co-Coach Engine achieve}

3D Sport Training Co-Coach Engine including the realization of three-dimensional motion simulation, the actual situation contrast, action choreography, the action editor, kinematics analysis, video analysis and other six kinds of auxiliary training methods, in the back of the upgrade version, will join kinetic analysis. These methods can help athletes understand and master the essentials of sports action to help athletes understand the nature of sports action from the physiological and physical laws, and according to its own conditions, develop appropriate training measures. The actual situation by comparison module standard action motion video recording and sports simulation system virtual athlete will be the same with the screen contrast Perspective display, which can be intuitive, accurate comparison of the difference between the two, to help athletes improve athletic performance and training efficiency. Three-dimensional motion analysis technology analysis including kinematic and dynamic analysis. Analysis of trends such as a joint body of a rotation angle, analysis of different height, athletes and other differences between different joint lengths in the same action in order to quantify the way the image of the athlete's movement status display. We choose the focus of joint virtual athletes, a visual display of the center of gravity within a certain time trajectories shown in Figure 3.

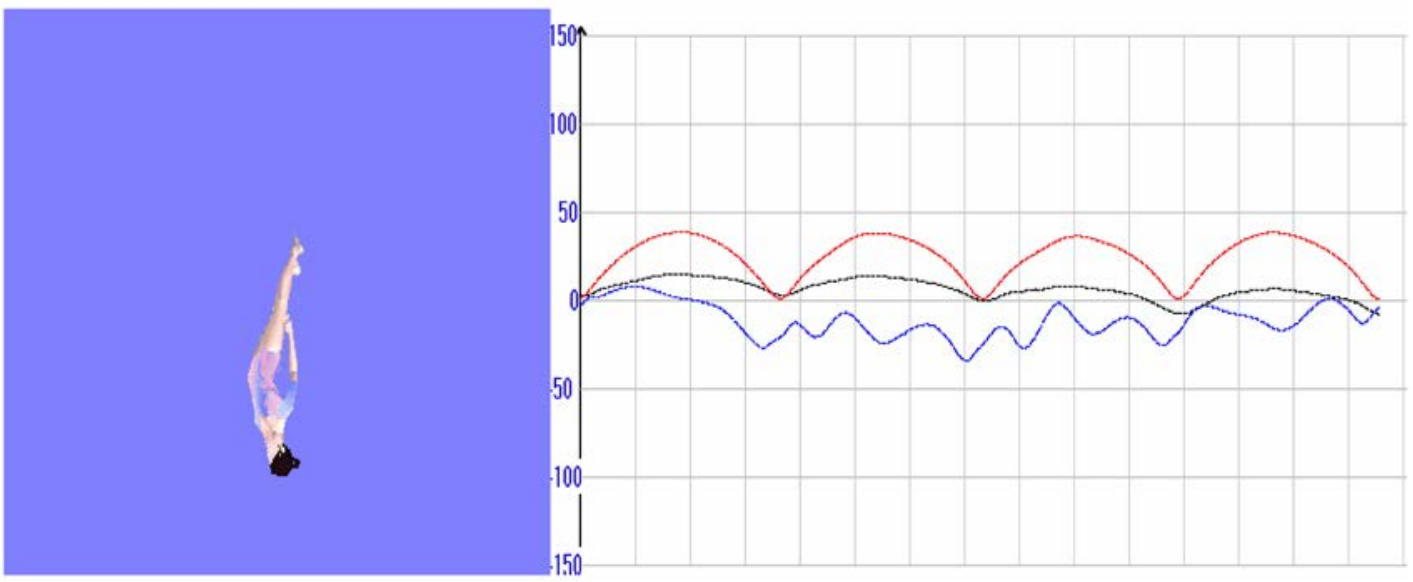

Figure 3. 3D virtual athletes focus locus 


\section{Computer-assisted three-dimensional sports training system analysis}

The system is based on real-time motion redirection human animation blending: First automatic extraction of three-dimensional model of three-dimensional skeleton connection diagram. Then some preprocessing operation skeleton model, so that you can complete with skeleton joint CMU-one match point; real-time acquisition Kinect motion data to automatically drive a three-dimensional model, combined with redirection technology to ensure realistic human motion characteristics.

Real-time animation synthesis system designed for dual-threaded, as shown in Figure 4 . The first thread to access real-time capture device motion capture human motion data. The second thread is responsible for loading 3D mesh, 3D mesh models and key point matching skeleton automatic extraction, skin deformation, and motion redirection. 1 thread 2 and thread to communicate and interact through a mutex variable data.

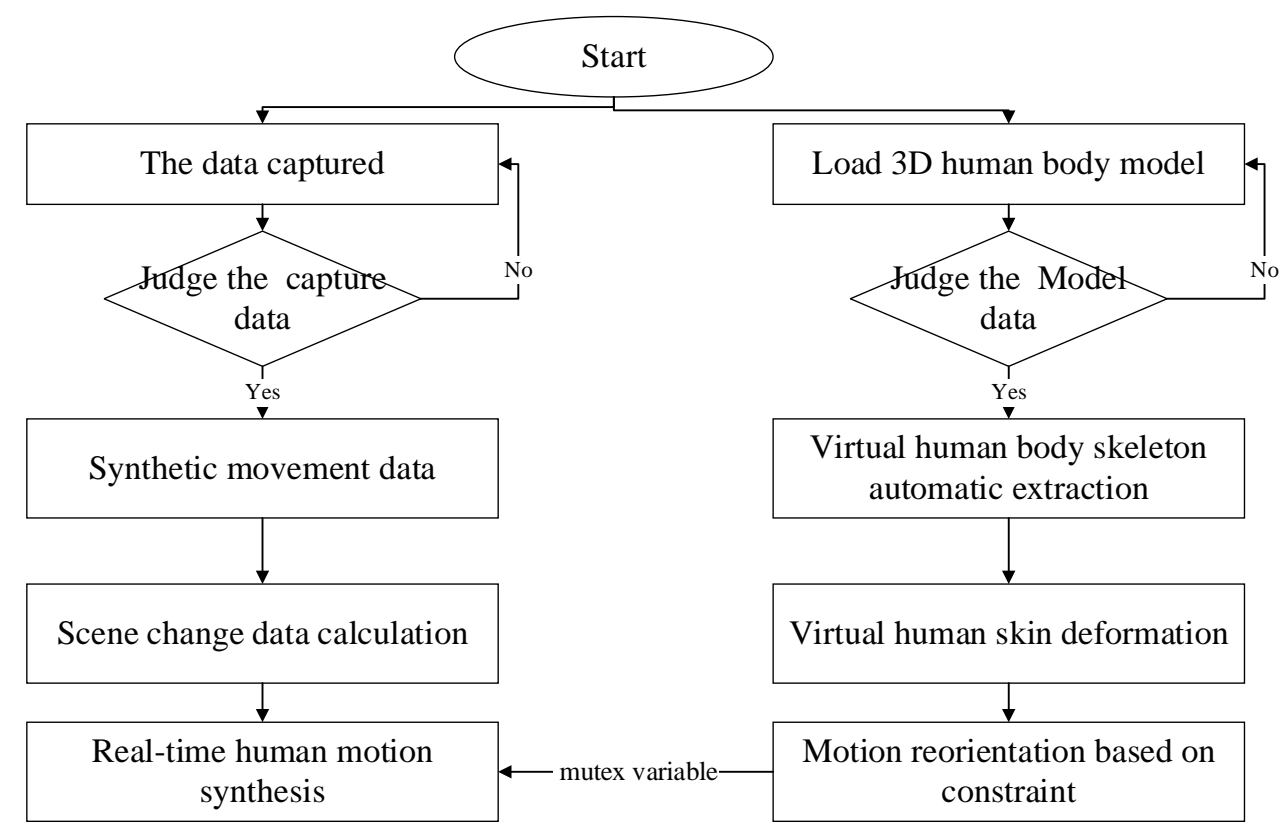

Figure 4. The design flow of Computer-assisted three-dimensional sports training system

The framework includes a number of low visa into high-dimensional data module, three-dimensional grid load, mesh skeleton extraction, matching and real-time motion capture data between nodes driven mesh model. The ultimate goal of the three-dimensional human motion in real-time motion capture system data-driven model, the system modules to achieve other three modules hint. Three-dimensional grid load is a fundamental part of the three modules.

System through the following steps to access the user low-dimensional motion signals into high-dimensional human motion data and accurate:

- According to the position and movement marks a recent synthesis for local sports capture database of choice;

- According to the selected local linear model to generate a motion, and then smoothing model for online motion synthesis;

- According to the root location in the face, the compound new mobile data network, it can be used for animation.

\section{Conclusion}

With the development of computer 3D technology, computer simulation of human motion analysis in the field of sports it has been widely used. The establishment of human motion model is carried out computer simulations of human movement key. However, modeling is an arduous and complicated task, the conventional method has its own characteristics and advantages, there are also their limitations. The combination of different research methods, use of their complementary 
strengths will be the focus and direction of research. Also with the introduction of artificial intelligence, human motion simulation by the mechanical characteristics of the individual worker to a person with a life simulation characteristics conversion, campaign workers, who will also be autonomous, intelligent behavior, and a plurality of artificial life the combination and coordination of behavior, people workers' behavior reflects the social property, which would be human motion simulation revolutionary change.

\section{Reference}

[1] Ferrari S, Carlomagno N, Di Tore P A, et al. How technologies in the classroom are modifying space and time management in teachers' experience?[J]. REM-RESEARCH ON EDUCATION AND MEDIA, 2013, 5(2): 79-90.

[2] Fogtmann M H, Grønbæk K, Ludvigsen M K. Interaction technology for collective and psychomotor training in sports[C]//Proceedings of the 8th International Conference on Advances in Computer Entertainment Technology. ACM, 2011: 13.

[3] Ochi Y, Takeda Y. Development of a Camera Control System Using Human Gesture Recognition[C]//Hong Kong: Proceedings of the International Multi Conference of Engineers and Computer Scientists. 2013.

[4] Wei Y, Yan H, Bie R, et al. Performance monitoring and evaluation in dance teaching with mobile sensing technology[J]. Personal and Ubiquitous Computing, 2014, 18(8): 1929-1939.

[5] Yamaoka K, Uehara M, Shima T, et al. Feedback of Flying Disc Throw with Kinect and its Evaluation[J]. Procedia Computer Science, 2013, 22: 912-920.

[6] Wattanasoontorn V, Magdics M, Boada I, et al. A kinect-based system for cardiopulmonary resuscitation simulation: a pilot study[M]//Serious Games Development and Applications. Springer Berlin Heidelberg, 2013: 51-63. 